\title{
Coherent wavepacket motion in an ultrafast electron transfer system monitored by femtosecond degenerate four-wave-mixing and pump-probe spectroscopy
}

Yutaka Nagasawa ${ }^{1-3 *}$, Yusuke Yoneda ${ }^{1}$, Shohei Nambu ${ }^{1}$, Masayasu Muramatsu ${ }^{1}$, Eisuke

Takeuchi $^{1}$, Hiroki Tsumori ${ }^{1}$, Soichiro Morikawa ${ }^{1}$, Tetsuro Katayama ${ }^{3}$, and Hiroshi

Miyasaka ${ }^{1,2 *}$

1. Division of Frontier Materials Science, Department of Materials Engineering Science,

Graduate School of Engineering Science, Osaka University, Toyonaka, Osaka

560-8531, Japan

2. Center for Quantum Science and Technology under Extreme Conditions, Osaka

University, Toyonaka, Osaka 560-8531, Japan

3. PRESTO, Japan Science and Technology Agency (JST), Kawaguchi, Saitama

332-0012, Japan 


\begin{abstract}
Coherent nuclear wavepacket motions were monitored by three types of femtosecond time-resolved spectroscopy, namely, transient absorption measurement utilizing white-light supercontinuum (WC-TA), degenerate four-wave-mixing (DFWM), and pump-probe (PP) measurements, for an ultrafast intermolecular electron transfer (ET) system with a dye molecule, oxazine $1(\mathrm{Ox} 1)$, dissolved in an electron donating solvent, N,N-dimethylaniline (DMA). Vibrational frequencies of the wavepacket motion in the excited and in the ground states were 560-562 and 567-569 $\mathrm{cm}^{-1}$, respectively, with only a few frequency difference of 5-9 $\mathrm{cm}^{-1}$, which were clearly distinguishable by the highly accurate measurements. In DMA, the excited state wavepacket motion declined with time constant of 160-240 fs which is somewhat longer than that of the ultrafast ET; 60-80 fs.
\end{abstract}

\title{
Keywords
}

ultrafast spectroscopy; electron transfer; coherent nuclear wavepacket motion; degenerate four-wave-mixing 


\section{Introduction}

Chemical reaction is a process that transforms the molecular structure of a compound into another, thus, certain molecular motions are considered to be involved in a specific reaction. For a simple two atomic molecule, it is easy to imagine that the stretching of a covalent bond between the atoms is coupled to a photo-dissociation reaction. In the case of sodium iodide, Zewail and coworkers have observed by femtosecond ultrafast spectroscopy that the photoproduct, sodium and iodine atoms, are produced stepwise each time when the molecule is stretched[1,2]. However, in the case of more complex chemical reaction of polyatomic molecules, it becomes difficult to identify the molecular motion involved.

In the case of electron transfer (ET), the particle in motion is an electron, thus simply thinking, it may seem that it is independent from the motion of atoms and molecules. However, solvent reorganization (solvation) is considered to be the driving force of ET in polar solvents because the charge or electric dipole is significantly stabilized by the polarization induced by solvation[3-8]. Moreover, when electron moves from one molecular orbital to another, molecular structure could also change as a 
consequence of the change in bonding ability. Therefore, the motion of solvent molecules and/or intramolecular atoms which are much heavier than an electron could be the rate limit factor for ET. In relation with the ET theory of Marcus[5,9], the effect of solvation upon ET have been studied extensively[6-8], while the effect of intramolecular nuclear motion remains mostly unexplored. It is considered that asymmetric bell-shaped energy gap dependence of ET arises from the contribution of vibrational levels in the product state[10,11], however, specific vibration involved in ET is yet to be identified.

From the view point of dynamics, intramolecular nuclear organization is considered to exceed the solvation process and induces ultrafast ET. One of such well studied system is a dye dissolved in neat electron donating solvents[12-17]. Because the electron acceptor is always surrounded by donors, the ET occurs for the most favorable pair without any molecular diffusion. By femtosecond ultrafast spectroscopies, this system is reported to exhibit ET with time constant of $<100 \mathrm{fs}[18,19]$ which is even shorter than the usual picosecond vibrational dephasing time. Especially, coherent nuclear wavepacket motions have been reported for oxazine 1 (Ox1) in N,N-dimethylaniline (DMA) by Zinth and coworkers[19-21], the structures of which are shown in Figure 1. However, the vibrational frequencies of the wavepackets did not 
change considerably upon photoexcitation nor ET, i.e., 562 and $567 \mathrm{~cm}^{-1}$ for the excited and ground states, respectively, and they were overlapped in a wide range of probe wavelengths. Thus, the time behavior of the wavepacket motion during the course of ET is not yet fully evaluated.

\section{Insert Figure 1 here.}

Recently, we have also started to investigate the details of the wavepacket motion in Ox1/DMA system by means of degenerate four-wave-mixing (DFWM) spectroscopy[22], and in the present article, two types of femtosecond pulse laser spectroscopies[23-27], namely transient absorption utilizing white-light supercontinuum (WC-TA) and pump-probe (PP) techniques, are also been applied to extract the behavior of wavepacket motions. WC-TA spectroscopy can simultaneously monitor the entire visible range of $400-800 \mathrm{~nm}$, although, higher $\mathrm{S} / \mathrm{N}$ ratio and time-resolution can be attained by DFWM and PP spectroscopies carried out at a single wavelength. Background-free three-pulse DFWM can also coherently control wavepacket motions[28,29] and, it is reported that coherent vibrations can be selectively switched from that in the ground state to that in the excited state in the case of neat iodine vapor[30,31]. Tuning the pump and probe wavelengths will also enable access to the 
details of the wavepacket dynamics. It would be an interesting task to distinguish the wavepackets of Ox1 with similar frequencies in multiple electronic states and extract the real time behavior of the one involved in the ET process by combination of these spectroscopies.

\section{Experimental methods}

Setup for ultrafast measurements have been reported previously [32,33], although the laser system was replaced by non-collinear optical parametric amplifier (TOPAS-White, Light Conversion) pumped by regenerative amplified Ti: sapphire laser (Solstice or Tsunami with Spitfire, Spectra Physics). For WC-TA measurement, pulse centered at ca. $660 \mathrm{~nm}$ generated from TOPAS-White and white-light supercontinuum generated from

a $1 \mathrm{~mm}$ glass plate were utilized and the polarization was set at magic angle. The signal and the reference pulses were detected with multichannel diode array systems (PMA-10, Hamamatsu) and sent to a personal computer for further analysis. Spectra were calibrated for group velocity dispersion by optical Kerr measurement between the pump pulse and the white-light continuum. The energy of the pump pulse measured at the sample position was ca. $10 \mathrm{~nJ}$ and the size of the laser beam was ca. $0.15 \mathrm{~mm}$ at the 
focusing point. The fwhm of the pump pulse was determined to be ca. 18 fs while that of the supercontinuum depends on the wavelength. Consequently, the time resolution of the apparatus depends on the wavelength and it was ca. $30 \mathrm{fs}$ around $650 \mathrm{~nm}$ while it stretches to $45 \mathrm{fs}$ around $450 \mathrm{~nm}$.

For degenerate four-wave-mixing (DFWM) measurement, pulses generated at wavelengths centered at ca. $620 \mathrm{~nm}$ or $660 \mathrm{~nm}$ were divided into three beams by reflective neutral density (ND) filters. There are two variable time delays that can be controlled for DFWM measurement, i.e. the interval between the first and the second pulse, $t_{12}$ and that between the first and the third pulse, $t_{13}$. In the present case, $t_{12}$ was set at certain values such as $-13,0$, and 13 fs and $t_{13}$ was scanned. The pulse duration and pulse energies measured at the sample position were 12-14 fs and 4-7 nJ, respectively, and the size of the laser beam was ca. $0.1 \mathrm{~mm}$ at the focusing point. For PP measurement, only two of the three pulses were utilized and the energy of the probe pulse was reduced to ca. $0.5 \mathrm{~nJ}$ by a variable ND filter. The signals were measured by photodiodes with lock-in amplifiers (EG\&G Princeton Applied Research, Model 5210).

Oxazine $1(\mathrm{Ox} 1)$ was purchased from Exciton Chemicals, N,N-dimethylaniline (DMA) and 1-chloronaphthalene (1-CN) were from Aldrich (redistilled, 99.5\%+) and 
Tokyo Chemical Industry (EP, $>97.0 \%$ ), respectively, and used without further purification. Absorption and fluorescence spectra were measured by Hitachi U-3500 spectrophotometer and F850 spectrofluorometer, respectively.

\section{Results and discussion}

\section{3-1. Steady state electronic spectra}

The absorption spectra of Ox1 in DMA and in an inert solvent, 1-CN, are shown in

Figure 2a. Because the polarity of $1-\mathrm{CN}$ is similar to that of DMA, i.e., the values of normalize empirical parameter of solvent polarity are 0.19 and 0.22 , respectively[34], the absorption maximum is located at similar wavelength of ca. $658 \mathrm{~nm}$ without any solvatochromic shift. It can be also seen that the spectrum in DMA is significantly broader than that in 1-CN, although no additional new band is detectable, which indicates that the solute and solvent are forming a weak charge transfer (CT) complex [35]. The fluorescence of Ox1/DMA was undetectable due to the quenching induced by the ultrafast excited state ET[15], thus, only the fluorescence spectrum of Ox1/1-CN is presented in Figure 2a. The fluorescence quantum yields of Ox1 in ethanol and 1,2-dichloroethane are reported to be 0.11 and 0.44 , respectively[36]. From these values, 
we estimate that that in $1-\mathrm{CN}$ to be ca. 0.49 . The lower quantum yield in the protic solvent could be due to enhancement of nonradiative decay by formation of hydrogen-bonding network with the solvent.

\section{Insert Figure 2 here.}

The laser spectrum was located at either ca. $620 \mathrm{~nm}$ or $660 \mathrm{~nm}$ with bandwidths of ca. $50 \mathrm{~nm}$, as can be seen in Figure 2b. For WC-TA measurement, the pulse at ca. $660 \mathrm{~nm}$ was employed, while for DFWM and PP measurements, both wavelengths were utilized. It is reported that the excited state absorption of Ox1 is overlapped with the ground state bleach in the range of $<640 \mathrm{~nm}$ [19]. Thus, excited state dynamics is expected to be observable for DFWM and PP measurements with probe pulse at $620 \mathrm{~nm}$ which is on the blue side of the absorption maximum. On the other hand, the laser pulse centered at $660 \mathrm{~nm}$ is directly in resonance with the maximum absorbance and somewhat overlapped with the fluorescence spectrum. Thus, ground state bleach accompanied by stimulated emission are expected to be observable in this wavelength region.

\section{3-2. WC-TA measurement}


In order to obtain an overview of the reaction, WC-TA measurement was carried out by probing the entire visible range of $400-750 \mathrm{~nm}$. The contour plot of WC-TA spectrum of $\mathrm{Ox} 1$ in 1-CN and in DMA in the range of 580-750 $\mathrm{nm}$ are shown in Figure 3 where the ground state bleach and stimulated emission can be observed as a negative absorbance difference, $\Delta_{\mathrm{abs}}$. Due to the ET in DMA, the negative $\Delta_{\mathrm{abs}}$ is significantly reduced in the longer wavelength side of the bleach (Figure 3b) indicating suppression of the stimulated emission compared to that in 1-CN (Figure 3a). It can be also seen that the WC-TA is immensely modulated by coherent wavepacket motions which can be either in the ground or in the excited state of Ox1/1-CN, while that in the ground state or the product state should be dominant in DMA where the excited state is immediately diminished by the ultrafast ET.

Insert Figure 3 here.

In the range of 400-600 $\mathrm{nm}$ shown in Figure 4a, excited state absorption bands of Ox1/1-CN can be observed as a positive $\Delta_{\mathrm{abs}}$ at ca. $450 \mathrm{~nm}$ and $560 \mathrm{~nm}$, the time dependence of which at $560 \mathrm{~nm}$ is shown in Figure 5a. Note that the band at $560 \mathrm{~nm}$ is modulated by a wavepacket motion in the excited state with a oscillation period of ca. 60 fs which corresponds to a frequency of ca. $560 \mathrm{~cm}^{-1}$. Zinth and coworkers have 
reported frequencies of $562 \pm 2 \mathrm{~cm}^{-1}$ and $602 \pm 3 \mathrm{~cm}^{-1}$ for the excited state wavepacket motion[19] which is only slightly shifted from those in the ground state, i.e., they were determined to be $567 \mathrm{~cm}^{-1}$ and $608 \mathrm{~cm}^{-1}$ by a resonance Raman scattering experiment[20].

In DMA, the excited state absorption bands are immediately taken over by a new band at ca. $430 \mathrm{~nm}$ as can be seen in Figure $4 \mathrm{~b}$ which is reported to be that of the reduced neutral radical form of Ox1 $\left(\mathrm{Ox} 1^{\circ}\right)[20,37]$. The absorption band of DMA cation $\left(\mathrm{DMA}^{+}\right)$is reported to peak at ca. $473-475 \mathrm{~nm}[38,39]$ which is not clearly observable in this case, presumably overlapped with that of $\mathrm{Ox} 1^{\circ}[20,37]$. At the peak wavelength of the excited state $(560 \mathrm{~nm}), \Delta_{\mathrm{abs}}$ turns negative and the wavepacket motion is no longer detectable (Figures 5a). At this wavelength, measurements were not reported by Zinth and coworkers[20]. Although they have reported wavepacket motion in the range of 440-500 nm, we were unable to detect any wavepacket at the peak wavelength of Ox 1 ' $(430 \mathrm{~nm})$ as shown in Figure $5 \mathrm{~b}$. This discrepancy is due to the different experimental methods employed. Zinth and coworkers utilized two-color PP measurements with pump and probe pulses centered at 630 and $465 \mathrm{~nm}$ with pulse durations of 9 and $14 \mathrm{fs}$, respectively. Meanwhile, we employed supercontinuum that covers the entire visible range of $400-800 \mathrm{~nm}$ as a probe. The chirp of the signal due to the group velocity 
dispersion can be estimated and compensated numerically by measuring the Kerr signal between the pump and the supercontinuum, while the duration of the supercontinuum at each wavelength is not easy to determine. From the rise of the WC-TA signal of Ox1/1-CN, the time-resolution was estimated to be ca. 30 and $45 \mathrm{fs}$ at $650 \mathrm{~nm}$ and 450 $\mathrm{nm}$, respectively. Thus, in the wavelength range of $<450 \mathrm{~nm}$, the time-resolution may not be sufficient enough to resolve the $560 \mathrm{~cm}^{-1}$ oscillation.

\section{Insert Figure 4 and 5 here.}

\section{3-3. DFWM measurement at $620 \mathrm{~nm}$}

DFWM measurement was carried out at $620 \mathrm{~nm}$ (Figure 6) where Zinth and coworkers have reported overlap of the excited state absorption with the ground state bleach[19]. The DFWM signals of Ox1 in 1-CN and DMA obtained by setting $t_{12}$ at -13 , 0, and 13 fs are shown in Figures 6. For Ox1/1-CN, a spike appears near the time origin when $t_{12}$ is tuned to positive delay ( $t_{12}=13 \mathrm{fs}$ ) and, with further increasing $t_{12}$, the spike becomes dominant (not shown). In Figure 7a, oscillatory parts of the signal of Ox1/1-CN are shown which were obtained by subtracting the decay components (in the case of $1-\mathrm{CN}$, it is nearly constant) from the signal. At a negative delay of $t_{12}=-13 \mathrm{fs}$, although the amplitude is weaker, it seems that the oscillation endure longer than those 
at either $t_{12}=0 \mathrm{fs}$ or $13 \mathrm{fs}$. By a least-squares-fitting, the dephasing time for the strongest vibrational mode at $562 \mathrm{~cm}^{-1}$ is obtained to be $1.3 \mathrm{ps}$ at $t_{12}=13 \mathrm{fs}$ and $1.5 \mathrm{ps}$ at $t_{12}=0 \mathrm{fs}$ while it is $2.8 \pm 0.1$ ps at $t_{12}=-13$ fs. These two effects are due to photon echo type of signal appearing at positive value of $t_{12}$ where the rephasing double sided Feynman diagrams can be drawn[32]. As the value of $t_{12}$ increases, contribution from the echo type signal increases which rapidly decays due to the ultrafast electronic dephasing. Simultaneously, slowly decaying transient grating type contribution decreases, and as a consequence, vibrational dephasing appears accelerated[22].

\section{Insert Figure 6 and 7 here.}

Although the DFWM signals of Ox1/1-CN exhibits strong oscillations with nearly no decay, that in DMA exhibits ultrafast decay, which is due to the solute-solvent ET, and the oscillations are significantly reduced. The signal could be fitted reasonably well with oscillating components, two decay components and a rise. The fitting parameters for the oscillations are listed in Table 1 and the time constants (the values in the parenthesis are relative intensities) for the decays and rise are 59 fs $(0.60), 1.8 \pm 0.2$ ps (0.40), and $1.0 \pm 0.2 \mathrm{ps}(-0.16)$, respectively. The fastest and the longest decay correspond to the ET and the back ET processes, respectively[18,19], while the $1.0 \mathrm{ps}$ 
rise component is presumably due to the conformational relaxation in the $\mathrm{CT}$ state which is reported for the first time. Because DFWM is a back-ground-free homodyne measurement, two fold of the longest time constant, ca. $3.6 \mathrm{ps}$, agrees with the previously reported value of $3.8 \mathrm{ps}$ for the back ET [19]. On the other hand, two fold of the shortest time constant, ca. $120 \mathrm{fs}$, is somewhat longer than the reported values of 30 fs and $80 \mathrm{fs}[18,19]$. This is because the slower decaying components are serving as a local oscillator for the faster ones which enables heterodyne detection (cross term between the two components can be detected). Therefore, the directly obtained value of $60 \mathrm{fs}$ is closer to the actual time constant of the ET. Zinth and coworkers have claimed that the ET occurs in a double step manner with time constants of 30 and 80 fs $[19,20]$. However, the 30 fs component was not observable in our case, because it was not separable from the ultrafast decay caused by the destructive interference of nuclear wavepacket motions.

Insert Table 1 here.

It can be seen from the oscillatory part of the signal in DMA (Figure 7b), obtained by subtracting the decay components, that the vibrational dephasing is accelerated by the ultrafast decay. For the most prominent mode at $572 \mathrm{~cm}^{-1}$, the time 
constant for the dephasing is $440 \pm 20$ fs (Table 1) which is significantly longer than that obtained for the decay caused by the ET. It seems as if the oscillation in the excited state is not diminished instantaneously by the ET but somewhat extends into the product state. If the excited state absorption is completely eliminated by the ET, the excited state vibration should not be observable. Thus, residual oscillation indicates overlap of the product absorption with the laser spectrum centered at $620 \mathrm{~nm}$ as discussed in the next section regarding the PP signal.

Fourier transformed spectra of the oscillations (normalized) in 1-CN and DMA with $t_{12}$ set at 0 fs are shown in Figure 11a. Real parts of the spectrum is represented because it preserves the phase information, i.e., Lorentzian-shape band represents cosine function, while a derivative of that represents $\pi / 2$ shifted sine function, and a negative band represents $\pi$ shifted function. As can be seen from Figure 11a, all the bands have positive peaks without derivative-type shape, thus the initial phase of the vibration must be all similar. The band at $562 \mathrm{~cm}^{-1}$ is the dominant mode in 1-CN and it is slightly shifted to a higher frequency of $569 \mathrm{~cm}^{-1}$ in DMA. For a similar xanthene dye, Nile blue, an intense mode was observed at $590 \mathrm{~cm}^{-1}$ in the resonance Raman spectrum and it was assigned to the ring-breathing mode.[40] This assignment could also apply for the strong mode at ca. $562-569 \mathrm{~cm}^{-1}$ for $\mathrm{Ox} 1$. The bands in 1-CN are very sharp due to the 
long dephasing time, while the band shapes in DMA do not resemble Lorentzian but they rather resemble combination of a sharp band located on top of a broader background with similar frequencies but with shorter and longer dephasing times, respectively. Thus, fitting was carried out utilizing a cosine function multiplied by double exponential decay functions and the dephasing times (relative intensity) for the band at $570 \mathrm{~cm}^{-1}$ in DMA were obtained to be $240 \pm 40 \mathrm{fs}(0.80)$ and $1.2 \pm 0.2 \mathrm{ps}(0.20)$. This result indicates that two independent oscillations are arising from multiple electronic states. However, as mentioned previously, the decay components could also serve as a local oscillator for the oscillating components. Thus, an ultrafast decay in the local oscillator could induce an superficial rapid dephasing of vibration.

\section{3-4. PP measurement at $620 \mathrm{~nm}$}

Because homodyne detected DFWM signal has complication with the cross-terms among decaying and oscillating components, heterodyne detected PP measurement, utilizing the probe beam as a stable local oscillator, was also carried out which is free of such complications. As shown in Figure 8a, while the PP signal of Ox1/1-CN strongly oscillates without any decay, ultrafast decay and reduction of the vibrational amplitude can be observed for Ox1/DMA. The time constants (relative intensities) of the decay 
components are $81 \mathrm{fs}(0.34), 560 \pm 30 \mathrm{fs}(-0.13)$, and $3.2 \mathrm{ps}(0.66)$ for Ox1/DMA where the shortest and the longest time constants are in good agreement with the previously reported values for the ET and back ET[18,19]. This result confirms that the reduction of the vibrational amplitude is not just a reflection of an ultrafast decay in the local oscillator but it represents actual diminishment of the vibration.

The oscillatory part of the signal obtained by subtracting the decay components are shown in Figure $8 \mathrm{~b}$ and the fitting parameters for the oscillation are listed in Table 2. Interestingly, the vibration of Ox1/DMA initially dephases very rapidly with a time constant of $160 \pm 40 \mathrm{fs}$, which was obtained by a simple least-squares-fitting with a fixed frequency of $562 \mathrm{~cm}^{-1}$ in the range of $<500 \mathrm{fs}$, while the disappeared vibration seems to reappear and endure for $>3$ ps. Real parts of the Fourier transformed spectra of the vibrations are shown in Figure 12a. The major band in 1-CN is located at $561 \mathrm{~cm}^{-1}$ which is consistent with that obtained by DFWM measurement. Interestingly, negative band appears at $567 \mathrm{~cm}^{-1}$ in DMA. Note that the band shape is not a derivative of a Lorentzian but it looks rather like a sharp negative band overlapped on top of a broader positive band. It could be that the long-lasting vibration in the ground state and the fast decaying one in the excited state are phase-shifted by $\pi$. 
Insert Figure 8 and Table 2 here.

The rapid decay observed in the PP signal shown in Figure $8 \mathrm{a}$ indicates decrease of transmitted photons. Concerning the laser spectrum centered at $620 \mathrm{~nm}$ (Figure 2b), it is not plausible to be overlapped significantly with the stimulated emission of Ox1. Thus, the decrease of transmitted photons must be due to the overlap with the product state absorption. If the oscillation continues in the product state to some extent with similar frequency and in-phase with the excited state one, while it is out-of-phase by $\pi$ with that in the ground state, the oscillation can interfere destructively with the ground state one. Presumably, the disappearance and reappearance of the oscillation can be due to such interference effect.

\section{3-5. DFWM and PP measurement at $660 \mathrm{~nm}$}

DFWM and PP measurements were also carried out at $660 \mathrm{~nm}$ where the ground state bleach is dominant and the results are presented in Figures 9 and 10, respectively. As same as those excited at $620 \mathrm{~nm}$, for $\mathrm{Ox} 1 / 1-\mathrm{CN}$, nearly no decay with strong oscillations are observed, while an ultrafast decay appears in DMA. The time constants for the decays in PP signal are $51 \mathrm{fs}(0.65)$ and $3.3 \mathrm{ps}(0.35)$ which are consistent with other results[18,19]. As can be seen from Figure $2 \mathrm{~b}$, the probe spectrum is quite broad, 
thus the signal may have multiple contributions from the ground, excited, and product states. The ultrafast decay can be due to either stimulated emission or product state absorption, while the contribution from the former is expected to be more significant in this wavelength range. However, the amplitude of the oscillation in DMA is not reduced as much as that at $620 \mathrm{~nm}$ excitation. Moreover, the dephasing time for the 567-569 $\mathrm{cm}^{-1}$ band in DMA observed by DFWM and PP measurements are $1.0 \mathrm{ps}$ and $1.8 \mathrm{ps}$, respectively, which are significantly longer than those observed with $620 \mathrm{~nm}$ excitation (Tables 1 and 2). Therefore, it can be concluded that, at the excitation wavelength of $660 \mathrm{~nm}$, the excited state oscillation is hardly observable while the ground state wavepacket motion is dominant.

\section{Insert Figure 9 and 10 here.}

Real parts of the Fourier transformed spectra of the oscillations observed in DFWM and PP signals with 620 and 660 excitation are displayed in Figures 11 and 12. From these figures, one can notice that the frequency of the dominant band is usually at $567-569 \mathrm{~cm}^{-1}$ which is slightly shifted to lower frequency of $561-562 \mathrm{~cm}^{-1}$ only in 1-CN at $620 \mathrm{~nm}$ excitation where the excited state absorption is overlapped with the ground state bleach. Because in DMA, where the excited state population is immediately 
diminished within $100 \mathrm{fs}$, the frequency of $567-569 \mathrm{~cm}^{-1}$ can be assigned to the oscillation in the ground state and/or to that in the product state, while that at 561-562 $\mathrm{cm}^{-1}$ can be assigned to that in the excited state.

\section{Insert Figure 11 and 12 here.}

To confirm that this small frequency shift is not just an experimental error but it represents two distinct vibrational modes that originate from different electronic states, oscillatory components of the PP signal for Ox1/1-CN excited at $620 \mathrm{~nm}$ and $660 \mathrm{~nm}$ are compared in Figure 13. In the time range of $\leq 0.5 \mathrm{ps}$, the oscillations at both excitation wavelengths are in-phase, while, after $2.0 \mathrm{ps,} \mathrm{they} \mathrm{became} \mathrm{nearly}$ out-of-phase by $\pi$. The red dotted curves overlapped on the oscillations at $620 \mathrm{~nm}$ and $660 \mathrm{~nm}$ excitation are cosine functions with frequencies of 560 and $567 \mathrm{~cm}^{-1}$, respectively, that decay with time constant of $3.0 \mathrm{ps}$. The oscillation periods of 560 and $567 \mathrm{~cm}^{-1}$ modes are 59.6 and $58.8 \mathrm{fs}$, respectively, with only a difference of $0.8 \mathrm{fs}$. However, after 40 cycles of oscillation, this difference will become ca. 30 fs which is detectable by the current experimental time resolution. This result confirms that the frequency difference of only ca. $7 \mathrm{~cm}^{-1}$, originally reported by Zinth and 
coworkers[19,20], is not an error but real. Such a distinction is only made possible by the high accuracy of the current DFWM and PP measurements.

Insert Figure 13 here.

\section{Conclusions}

Two types of coherent wavepacket motion with frequencies of $567-569 \mathrm{~cm}^{-1}$ and 561-562 $\mathrm{cm}^{-1}$ were observed by means of femtosecond time-resolved spectroscopies (WC-TA, DFWM, and PP measurements) and they were assigned to the ground and excited state vibrations of $\mathrm{Ox} 1$, respectively. This conclusion was derived from the following observations; (i) The oscillation with frequency of $561-562 \mathrm{~cm}^{-1}$ was only observable in 1-CN at the excitation wavelength of $620 \mathrm{~nm}$ where the excited state absorption is overlapped with the ground state bleach. (ii) The $561-562 \mathrm{~cm}^{-1}$ oscillation was quickly diminished by the ET in DMA (decay time constant of 160-240 fs) and replaced by a weak oscillation at $567-569 \mathrm{~cm}^{-1}$. (iii) By comparing the time dependence of the vibrational phase in 1-CN at $620 \mathrm{~nm}$ and $660 \mathrm{~nm}$ excitation, it was confirmed that the frequency difference is indeed real. Such a distinction is only capable by femtosecond DFWM and PP measurements with high accuracy. 
The dephasing time in DMA for the excited state vibrational mode (160-240 fs)

is somewhat longer than the decay time of the ET (ca. 60-80 fs). It seems as if the oscillation is not diminished instantaneously by the ET but somewhat extends into the product state. However, we were unable to clearly distinguish the oscillation in the product state from that in the ground state. In the present experiment, the probe wavelength for DFWM and PP measurements were only limited to ca. $620 \mathrm{~nm}$ and 660 $\mathrm{nm}$. The entire spectral range must be scanned to thoroughly understand the role of coherent wavepacket motions in ET which will be our future project.

\section{Acknowledgement}

YN and TK were supported by Research for Embryonic Science and Technology (PRESTO), Japan Science and Technology Agency (JST). MM was supported by the fellowship (DC2) from Japan Society for the Promotion of Science (JSPS). HM was supported by a Grant-in-Aid for Scientific Research (23245004) from the Ministry of Education, Culture, Sports, Science and Technology (MEXT), Japan. 


\section{References}

[1] T.S. Rose, M.J. Rosker, A.H. Zewail, J. Chem. Phys. 88 (1988) 6672

[2] A.H. Zewail, J. Phys. Chem. A 104 (2000) 5660.

[3] P.F. Barbara, T.J. Thomas J. Meyer, M.A. Ratner, J. Phys. Chem. 100 (1996) 13148.

[4] H. Heitele, Angew. Chem. Int. Ed. Engl. 32 (1993) 359.

[5] R.A. Marcus, Rev. Mod. Phys. 65 (1993) 599.

[6] M. Maroncelli, J. Macinnis, G.R. Fleming, Science 243 (1989) 1674.

[7] M.L. Horng, J.A. Gardecki, A. Papazyan, M. Maroncelli, J. Phys. Chem. 99 (1995) 17311.

[8] S.J. Rosenthal, J. Jimenez, G.R. Fleming, J. Mol. Liq. 60 (1994) 25.

[9] R.A. Marcus, Annu. Rev. Phys. Chem. 15 (1964) 155.

[10] J. Jortner, M. Bixon, J. Chern. Phys. 88 (1988) 167.

[11] J. Ulstrup, J. Jortner, J. Chem. Phys. 63 (1975) 4358.

[12] T. Kobayashi, Y. Takagi, H. Kandori, K. Kemnitz, Y. Yoshihara, Chem. Phys. Lett. 180 (1991) 416.

[13] Y. Nagasawa, A.P. Yartsev, K. Tominaga, P.B. Bisht, A.E. Johnson, K. Yoshihara, J. Phys. Chem. 99 (1995) 653.

[14] Y. Nagasawa, A.P. Yartsev, K. Tominaga, A.E. Johnson, K. Yoshihara, J. Am. Chem. Soc. 115 (1993) 7922.

[15] Y. Nagasawa, A.P. Yartsev, K. Tominaga, A.E. Johnson, K. Yoshihara, J. Chem. Phys. 101 (1994) 5717.

[16] A. Yartsev, Y. Nagasawa, A. Douhal, K. Yoshihara, Chem. Phys. Lett. 207 (1993) 546.

[17] K. Yoshihara, K. Tominaga, Y. Nagasawa, Bull. Chem. Soc. Jpn. 68 (1995) 697.

[18] I.V. Rubtsov, H. Shirota, K. Yoshihara, J. Phys. Chem. A 103 (1999) 1801.

[19] M. Seel, S. Engleitner, W. Zinth, Chem. Phys. Lett. 275 (1997) 363.

[20] S. Engleitner, M. Seel, W. Zinth, J. Phys. Chem. A 103 (1999) 3013.

[21] B. Wolfseder, L. Seidner, W. Domcke, G. Stock, M. Seel, S. Engleitner, W. Zinth, Chem. Phys. 233 (1998) 323.

[22] Y. Nagasawa, Y. Yoneda, S. Nambu, M. Muramatsu, E. Takeuchi, H. Tsumori, H. Miyasaka, Vibrational Spectroscopy 70 ( 2014) 58.

[23] G.R. Fleming, Chemical applications of ultrafast spectroscopy, Oxford University Press, USA, 1986. 
[24] A.H. Zewail, Femtochemistry: Ultrafast Dynamics of the Chemical Bond, World Scientific, Singapore, 1994.

[25] M.H. Vos, J.-L. Martin, Biochim. Biophys. Acta 1411 (1999) 1.

[26] N. Tamai, H. Miyasaka, Chem. Rev. 100 (2000) 1875.

[27] M. Glasbeek, H. Zhang, Chem. Rev. 104 (2004) 1929.

[28] Y. Nagasawa, Y. Mori, Y. Nakagawa, H. Miyasaka, T. Okada, J. Phys. Chem. B 109 (2005) 11946.

[29] W.P. de Boeij, M.S. Pshenichnikov, D.A. Wiersma, J. Chem. Phys. 105 (1996) 2953.

[30] E.J. Brown, Q. Zhang, M. Dantus, J. Chem. Phys. 110 (1999) 5772

[31] B.I. Grimberg, V.V. Lozovoy, M. Dantus, S. Mukamel, J. Phys. Chem. A 106 (2002) 697.

[32] Y. Nagasawa, J. Photochem. Photobio. C 12 (2011) 31.

[33] Y. Nagasawa, K. Fujita, T. Katayama, Y. Ishibashi, H. Miyasaka, T. Takabe, S. Nagao, S. Hirota, Phys. Chem. Chem. Phys. 12 (2010, , ) 6067.

[34] C. Reichardt (Ed.)^(Eds.), Molecular Interactions. John Wiley \& Sons, 1982.

[35] S. Schneider, W. Stammler, R. Bierl, W. Ja"ger, Chem. Phys. Lett. 219 (1994) 433.

[36] R. Sens, K.H. Drexhage, J. Luminescence 24/25 (1981) 709.

[37] K. Yoshihara, Y. Nagasawa, A. Yartse, S. Kumazaki, H. Kandor, A.E. Johnson, K. Tominaga, Photochem. Photobiol. A 80 (1994) 169.

[38] T. Shida, Electronic Absorprion Spccrra of Radical Ions, Elsevier, Amsterdam, 1988.

[39] H. Kandori, K. Kemnitz, Y. Yoshihara, J. Phys. Chem. 96 (1992) 8042.

[40] M.K. Lawless, R.A. Mathies, J. Chem. Phys. 96 (1992) 8037 
Table 1. Fitting parameters for the vibrations observed in DFWM signal of Ox 1 with $t_{12}$ set at $0 \mathrm{fs}$.

\begin{tabular}{ccccc}
\hline \multirow{2}{*}{ Solvent } & $\lambda_{\mathrm{ex}} / \mathrm{nm}$ & $v_{1} / \mathrm{cm}^{-1}\left(A_{\mathrm{v} 1}\right)$ & $v_{2} / \mathrm{cm}^{-1}\left(A_{\mathrm{v} 2}\right)$ & $v_{3} / \mathrm{cm}^{-1}\left(A_{\mathrm{v} 3}\right)$ \\
& & $t_{\mathrm{v} 1} / \mathrm{fs}$ & $t_{\mathrm{v} 2} / \mathrm{fs}$ & $t_{\mathrm{v} 3} / \mathrm{fs}$ \\
\hline \multirow{3}{*}{$1-\mathrm{CN}$} & $261(0.19)$ & $563(0.58)$ & $608(0.23)$ \\
& & $920 \pm 60$ & 1500 & 1400 \\
& \multirow{2}{*}{660} & $262(0.23)$ & $567(0.56)$ & $607(0.21)$ \\
& & $770 \pm 50$ & 1700 & $1500 \pm 100$ \\
\multirow{2}{*}{ DMA } & $261^{\mathrm{a}}(0.24)$ & $572(0.33)$ & $615(0.43)$ \\
& \multirow{2}{*}{660} & 150 & $440 \pm 20$ & $200 \pm 10$ \\
& & $262(0.40)$ & $569(0.27)$ & $612(0.33)$ \\
& & 210 & 1000 & $290 \pm 10$
\end{tabular}

${ }^{\text {a }}$ The frequency was fixed.

Table 2. Fitting parameters for the vibrations observed in PP signal of Ox1.

\begin{tabular}{ccccc}
\hline \multirow{2}{*}{ Solvent } & \multirow{2}{*}{$\lambda_{\mathrm{ex}} / \mathrm{nm}$} & $\begin{array}{c}v_{1} / \mathrm{cm}^{-1}\left(A_{v 1}\right) \\
t_{v 1} / \mathrm{fs}\end{array}$ & $\begin{array}{c}v_{2} / \mathrm{cm}^{-1}\left(A_{v 2}\right) \\
t_{\mathrm{v} 2} / \mathrm{fs}\end{array}$ & $\begin{array}{c}v_{3} / \mathrm{cm}^{-1}\left(A_{v 3}\right) \\
t_{v 3} / \mathrm{fs}\end{array}$ \\
\hline \multirow{2}{*}{$1-\mathrm{CN}$} & 620 & $257(0.13)$ & $560(0.55)$ & $602(0.31)$ \\
& & $1100 \pm 100$ & 1300 & $840 \pm 50$ \\
& 660 & - & $569(0.63)$ & $609(0.27)$ \\
& 620 & - & $2400 \pm 100$ & $1500 \pm 100$ \\
DMA & & $562^{\mathrm{b}}$ & - \\
& \multirow{2}{*}{660} & $267(0.34)$ & $567(0.44)$ & $609(0.22)$ \\
& & $280 \pm 20$ & 1800 & $1400 \pm 100$
\end{tabular}

${ }^{\mathrm{b}}$ The frequency was fixed and the fitting was only carried out in the range of $<500 \mathrm{fs}$. 

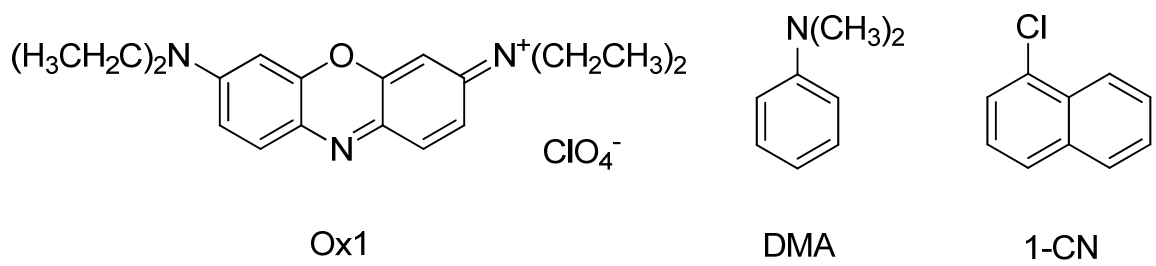

Figure 1. The molecular structures of oxazine 1 (Ox1), N,N-dimethylaniline (DMA), and 1-chloronaphthalene (1-CN). 

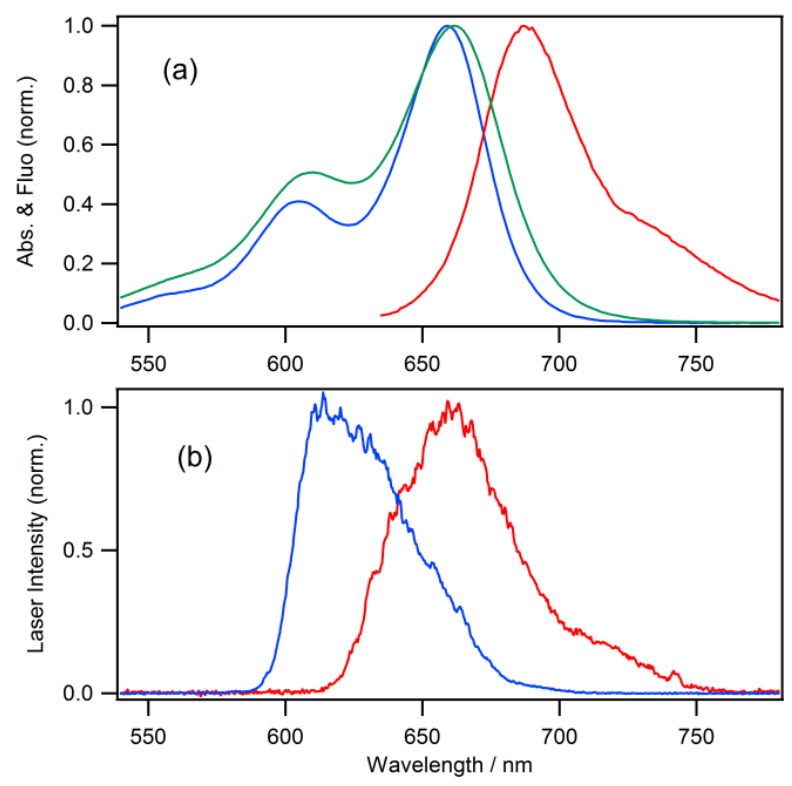

Figure 2. (a) The absorption spectra of $\mathrm{Ox} 1$ in 1-CN (green curve) and DMA (blue curve) and fluorescence spectrum in 1-CN (red curve). (b) Laser spectrum centered at ca. $620 \mathrm{~nm}$ (blue curve) and $660 \mathrm{~nm}$ (red curve). 

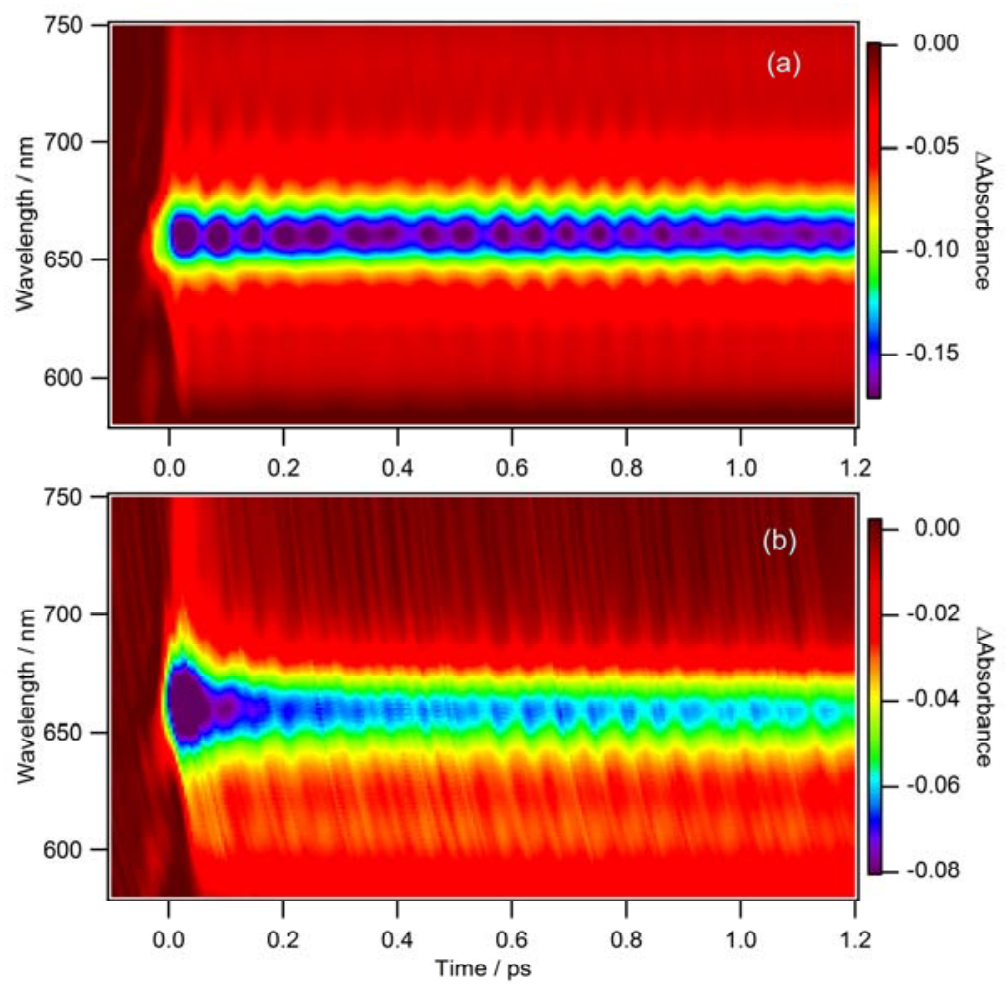

Figure 3. The contour plots of transient absorption (WC-TA) spectra of Ox1 in (a) $1-\mathrm{CN}$ and in (b) DMA in the wavelength range of 580-750 nm. 

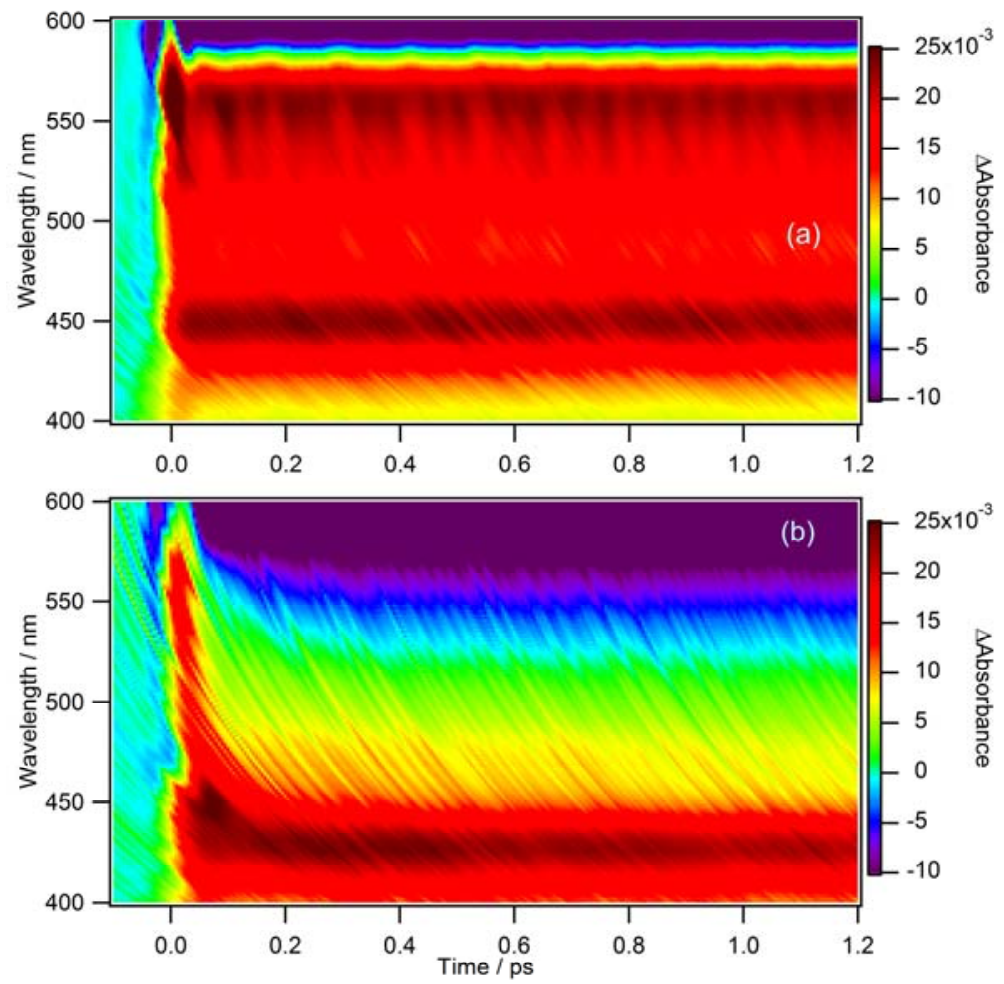

Figure 4. The contour plots of WC-TA spectra of Ox1 in (a) 1-CN and in (b) DMA in the wavelength range of 400-600 $\mathrm{nm}$. 

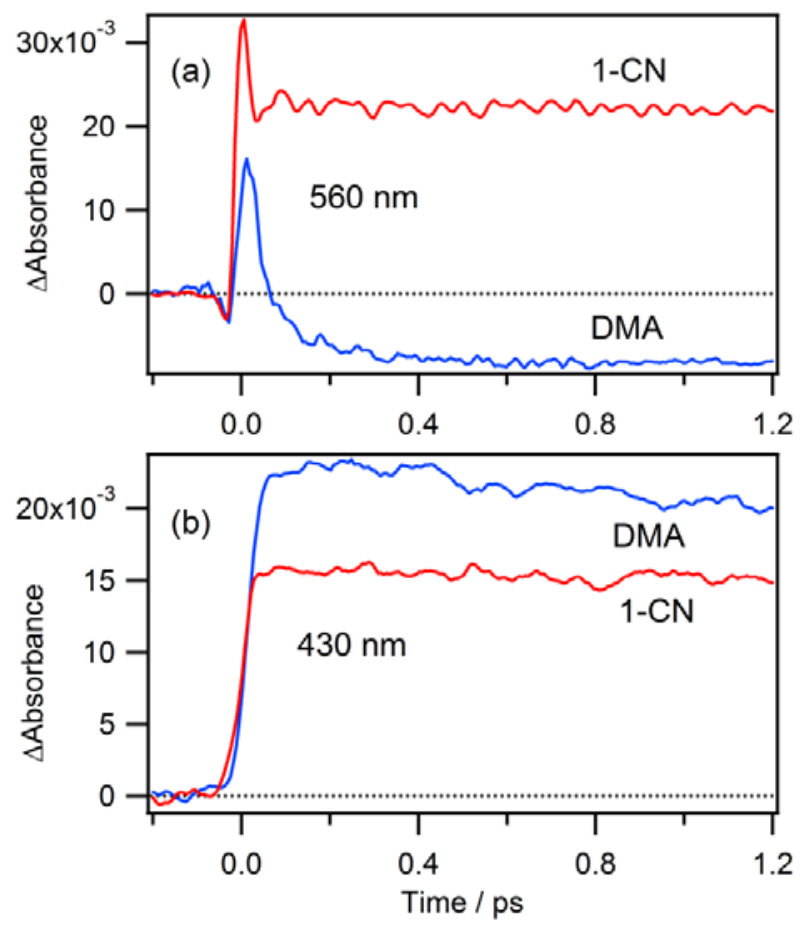

Figure 5. The time dependence of the differential absorbance at (a) $560 \mathrm{~nm}$ and (b) 430 $\mathrm{nm}$ of $\mathrm{Ox} 1$ in 1-CN and in DMA. 

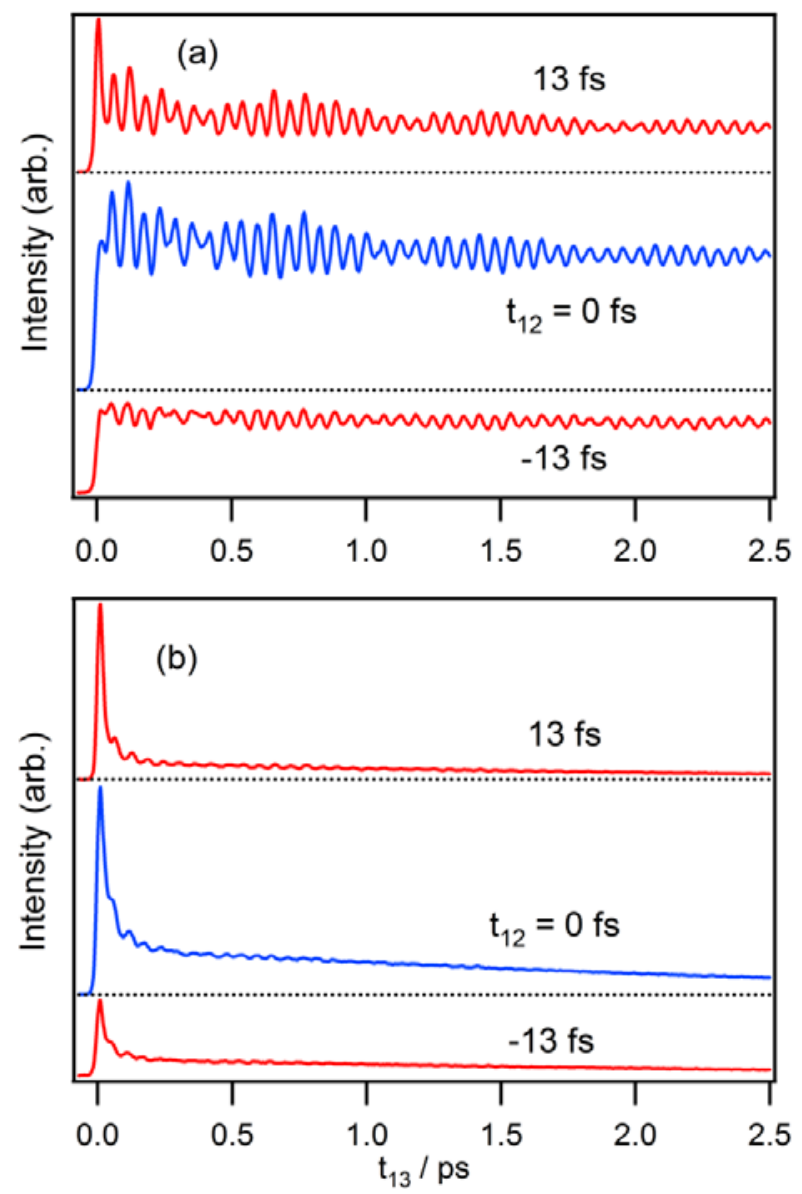

Figure 6. Femtosecond degenerate four-wave-mixing (DFWM) signals of Ox1 in (a) 1-CN and (b) in DMA excited at $620 \mathrm{~nm}$ with $t_{12}$ set at $-13,0$, and +13 fs and the phase-matching direction set at $-\boldsymbol{k}_{1}+\boldsymbol{k}_{2}+\boldsymbol{k}_{3}$. 

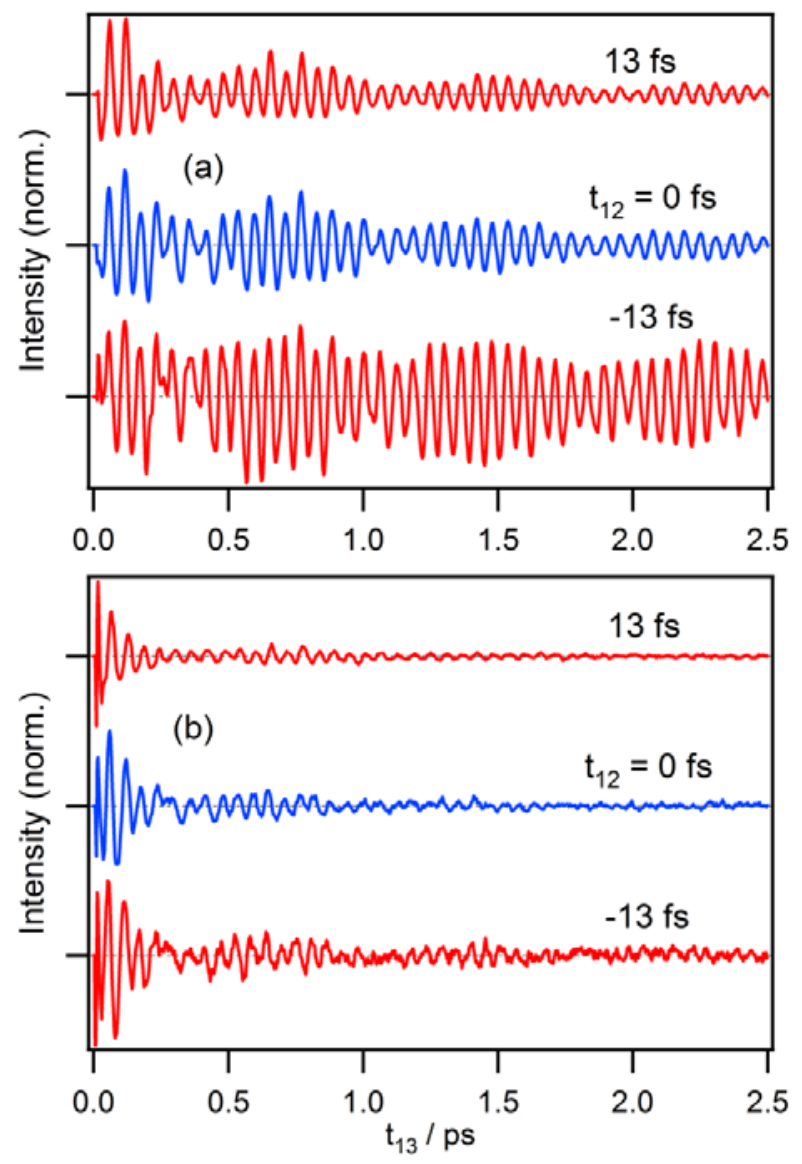

Figure 7. Oscillatory parts (normalized) of the DFWM signals of obtained by subtracting the decay components from the signal. Ox1 in (a) 1-CN and (b) in DMA excited at $620 \mathrm{~nm}$ with $t_{12}$ set at $-13,0$, and +13 fs and the phase-matching direction set at $-\boldsymbol{k}_{1}+\boldsymbol{k}_{2}+\boldsymbol{k}_{3}$. 

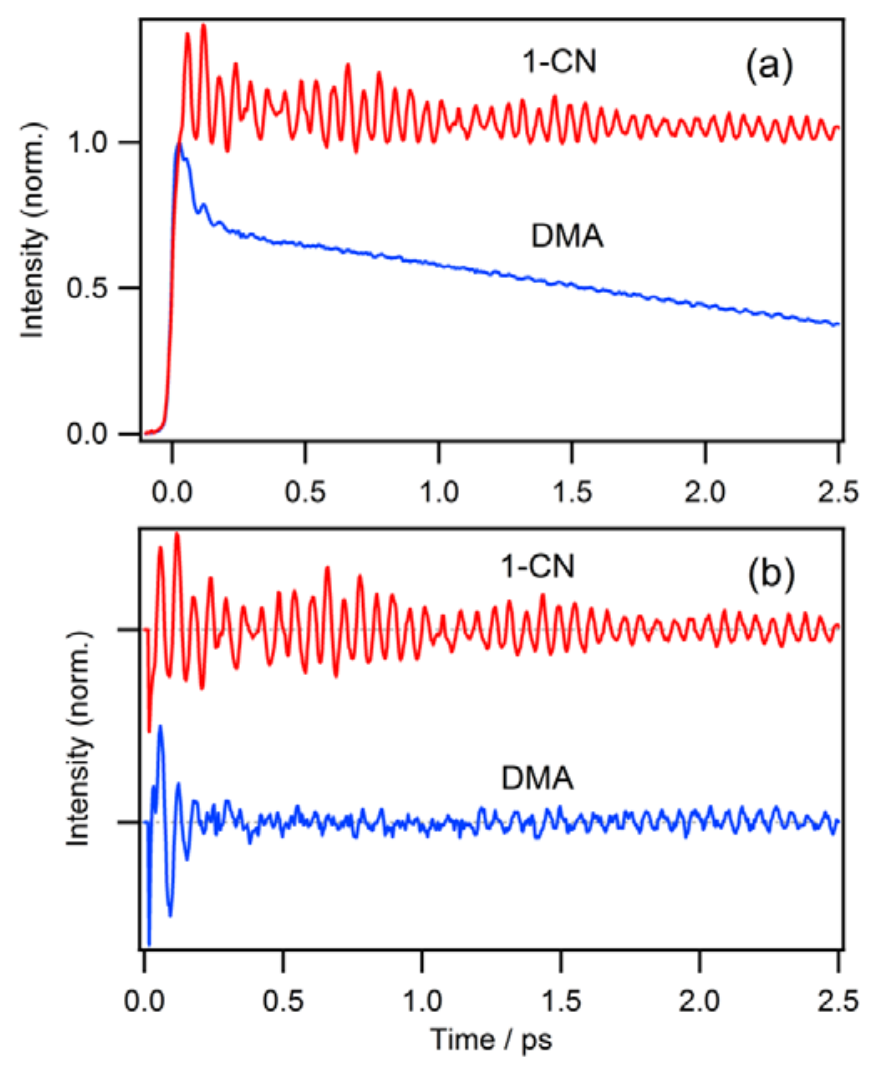

Figure 8. (a) Femtosecond pump-probe (PP) signals of Ox1 in 1-CN and DMA excited at $620 \mathrm{~nm}$. (b) Oscillatory parts (normalized) of the PP signals obtained by subtracting the decay components from the signal. 

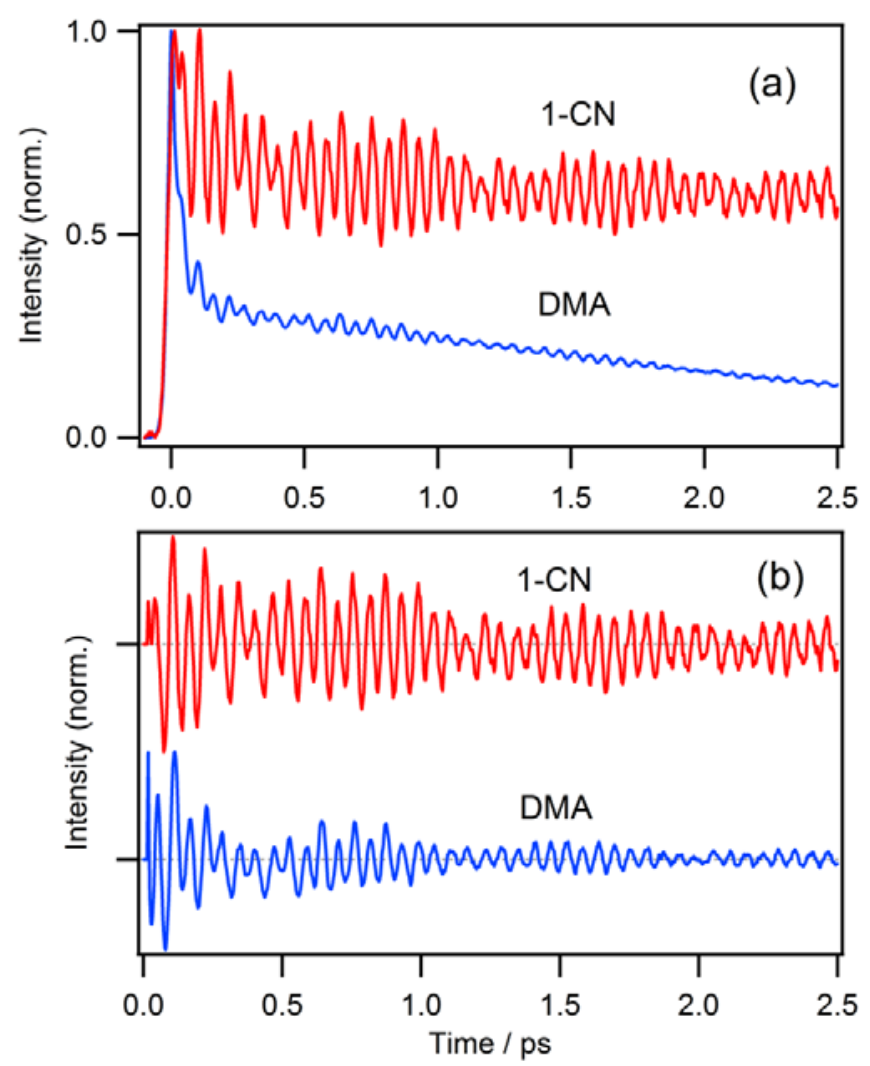

Figure 9. (a) Femtosecond DFWM signals (normalized) of Ox1 in 1-CN and DMA excited at ca. $660 \mathrm{~nm}$ with $t_{12}$ set at 0 fs. (b) Oscillatory parts (normalized) of the DFWM signals obtained by subtracting the decay components from the signal. 

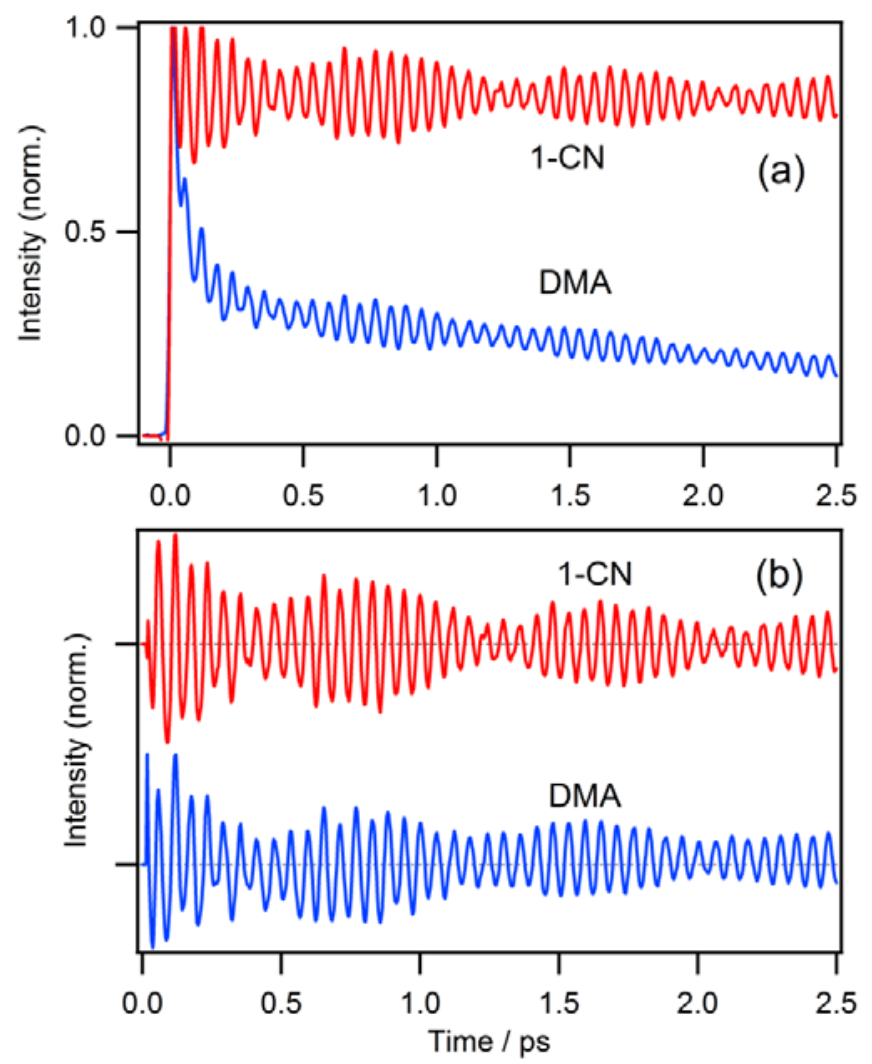

Figure 10. (a) Femtosecond PP signals (normalized) of $\mathrm{Ox} 1$ in $1-\mathrm{CN}$ and DMA excited at ca. 660nm. (b) Oscillatory parts (normalized) of the PP signals obtained by subtracting the decay components from the signal. 


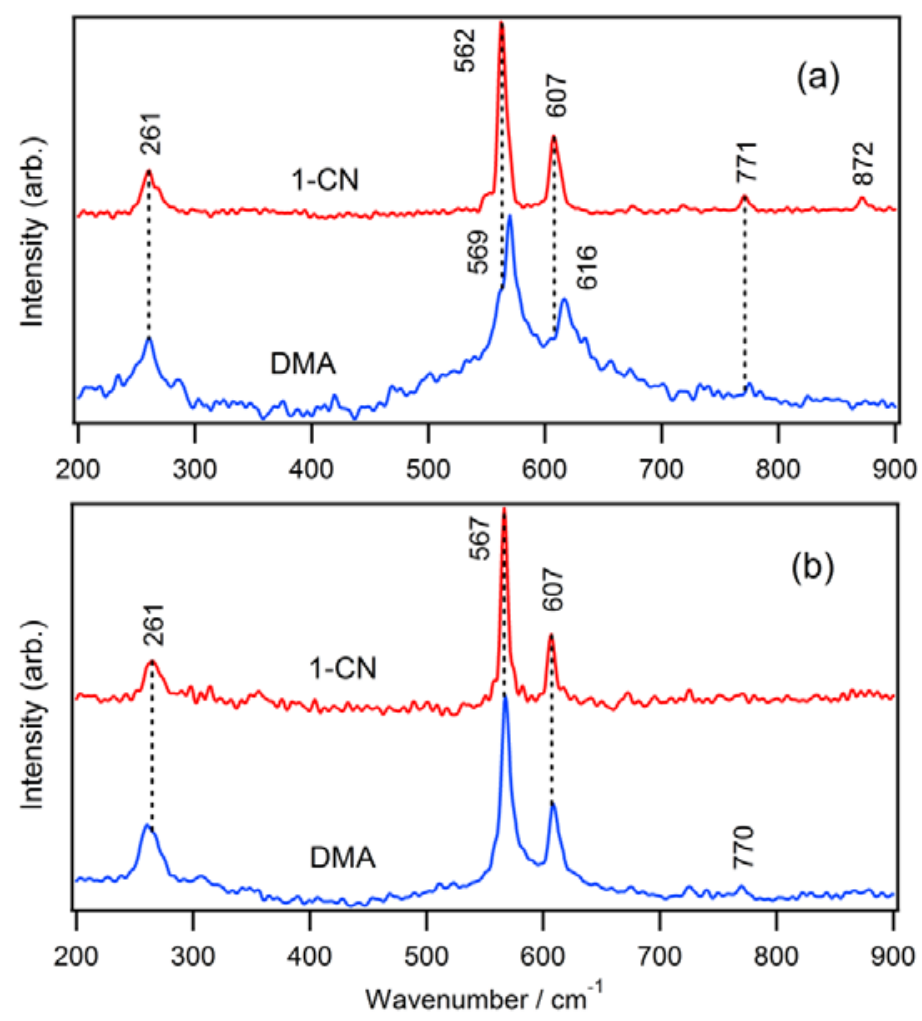

Figure 11. Real parts of the Fourier transformed spectra of the oscillations of DFWM signals of Ox1 in 1-CN (red curve) and DMA (blue curve) with $t_{12}$ set at 0 fs and center excitation wavelength of (a) ca. $620 \mathrm{~nm}$ and (b) $660 \mathrm{~nm}$, respectively. 

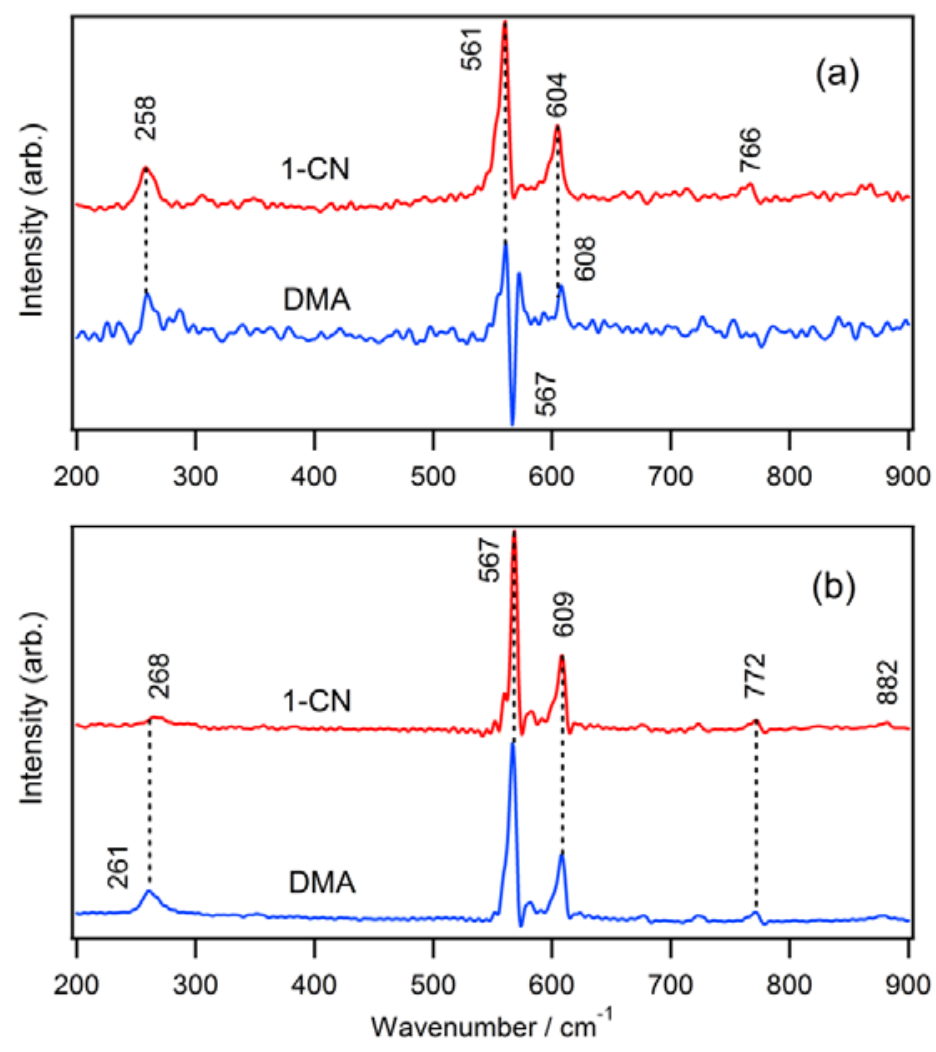

Figure 12. Real parts of the Fourier transformed spectra of the oscillations of PP signals of Ox1 in 1-CN (red curve) and DMA (blue curve) with center excitation wavelength of (a) ca. $620 \mathrm{~nm}$ and (b) $660 \mathrm{~nm}$. 


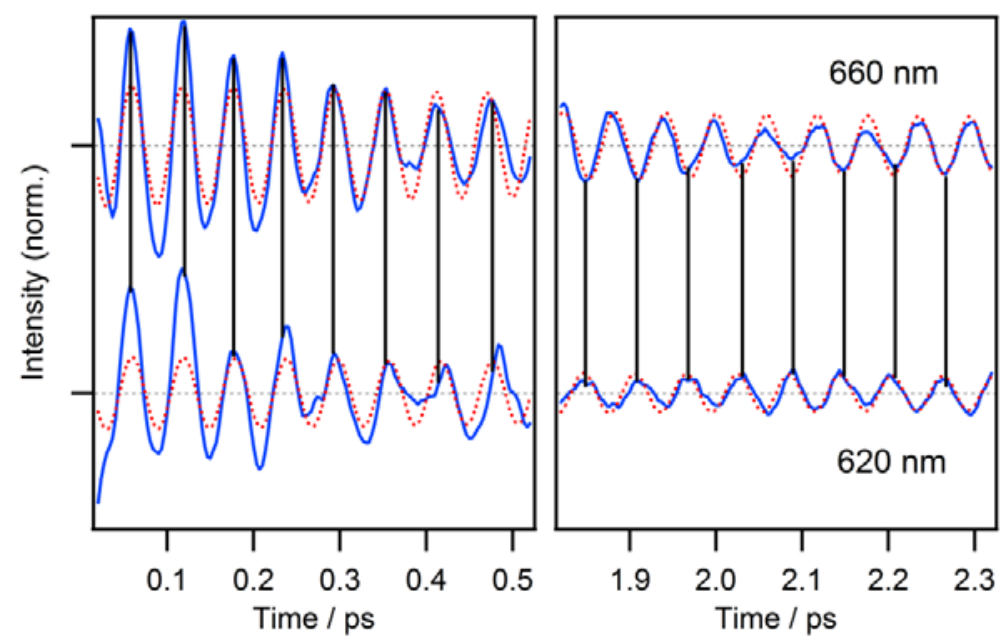

Figure 13. Oscillatory parts (normalized) of the PP signals for Ox1 in 1-CN excited at $620 \mathrm{~nm}$ and $660 \mathrm{~nm}$. Red dotted curves are exponentially decaying cosine functions with time constant of $3.0 \mathrm{ps}$ and frequencies of 560 and $567 \mathrm{~cm}^{-1}$, respectively. 
Graphical Abstract

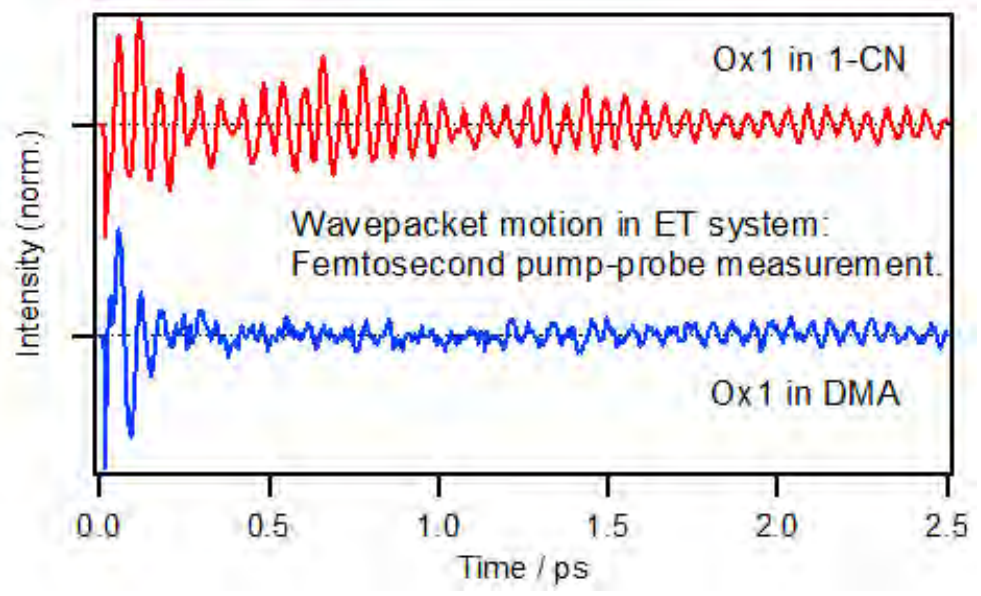

